\title{
Impacto de un Programa de Psicología Positiva en Sintomatologia Depresiva y Satisfaccion Vital en Adultos Mayores
}

\author{
Effects of a Positive Psychology Program in Depressive Symptoms \\ and Life Satisfaction in the Elderly
}

\author{
Alejandro Cuadra-Peralta ${ }^{*}$, , Constanza Veloso-Besio ${ }^{a}$, Giannina Puddu-Gallardo ${ }^{b}$, \\ Paulina Salgado-García ${ }^{a} \&$ Jenniffer Peralta-Montecinos ${ }^{a}$ \\ ${ }^{a}$ Universidad de Tarapacá, Arica, Chile \& ${ }^{b}$ Universidad Autónoma de Madrid, Madrid, España
}

\begin{abstract}
Resumen
La presente investigación estudia la efectividad de talleres basados en Psicología Positiva en adultos mayores, mediante el análisis de las variaciones pre-post del niveles de Satisfacción Vital y Sintomatología Depresiva. Se conformó tres grupos: el primero, un grupo cuasi experimental, basado en Psicología Positiva; el segundo, un grupo cuasi control activo, basado en actividades recreativas; y el tercero, un grupo cuasi control inactivo, sin intervención. Se utilizó la Escala de Depresión Geriátrica de Yesavage y Escala de Satisfacción Vital de Diener. Los resultados indican que sólo el grupo con taller basado en Psicología Positiva disminuye significativamente su Sintomatología Depresiva y aumenta significativamente sus niveles de Satisfacción Vital, luego de la intervención. Estos resultados se discuten a la luz de las investigaciones actuales en Psicología Positiva.

Palabras clave: Satisfacción vital, sintomatología depresiva, Psicología Positiva, felicidad.
\end{abstract}

\begin{abstract}
This research examines the effectiveness of workshops based on positive psychology for the elderly by analyzing pre-post changes in levels of life satisfaction and depressive symptomatology. Three groups were formed: the first, a quasi-experimental group based on positive psychology; the second, a quasiactive control group based on recreational activities; and the third, a quasi-inactive control group without intervention. The instruments used were: Yesavage Geriatric Depression Scale and Life Satisfaction Scale of Diener. Results indicated that only the group that attended positive psychology-based workshops significantly decreased depressive symptoms and significantly increased their levels of life satisfaction after the intervention. These results are discussed in the light of current researches in positive psychology.

Keywords: Life satisfaction, depressive symptoms, Positive Psychology, happiness.
\end{abstract}

Los cambios biológicos propios del envejecimiento, la presencia de déficits cognitivos, la coexistencia de otros problemas médicos y el uso de múltiples medicamentos, hace a los adultos mayores más vulnerables para presentar sintomatología depresiva (J. Martínez et al., 2002). De hecho, cabe destacar que la depresión es actualmente la patología más común en el segmento correspondiente a los adultos mayores (Departamento de Estadística e Información de Salud [DEIS], 2006). Debido a esto, y a la gran evolución demográfica de dicho grupo (Araujo \& Lobo Filho, 2009; Carneiro, Falcone, Clark, Z. Del Prette, $\&$ A. Del Prette, 2007), tanto a nivel mundial como na-

\footnotetext{
" Endereço para correspondência: Departamento de Psicología, Universidad de Tarapacá, 18 de Septiembre, 2222, Arica, Chile. E-mail: acuadra@uta.cl, veloso.cosntanza@gmail.com,ninapuddu@gmail.com, paulinasalgado@gmail.com e jperalta@uta.cl. Agradecimentos à Dirección de Investigación, Universidad de Tarapacá y Convenio desempeño MINUDUC - UTA.
}

cional, la depresión en los adultos mayores se ha vuelto un problema clínico complejo (Instituto Nacional de Estadísticas [INE], 2002) y de carácter prioritario en salud pública (Martín et al., 2008).

Se ha demostrado que el enfoque cognitivo conductual es útil y adaptable para tratar depresión en el adulto mayor, específicamente la terapia cognitiva de Beck (Contreras et al., 2006; Coon \& Thompson, 2003; Pérez \& García, 2001). Sin embargo, este tipo de tratamiento se enfoca sólo en la disminución de los síntomas negativos como criterio de mejora, marginando una característica central de la depresión como es la ausencia de emociones positivas, lo cual se relaciona tanto con la sintomatología como con el mantenimiento de dicha patología (B. Vera, 2006). Algunos investigadores han llamado la atención sobre dicha situación, por lo que están estudiando una nueva alternativa. Es así que A. Cuadra, Veloso, Ibergaray y Rocha (2010) y Seligman, Rashid y Parks (2006), plantean la existencia de un tratamiento diseñado desde la psicología positiva, el cual puede ser tan o incluso más 
eficiente que los tratamientos tradicionales para pacientes con depresión. Esta forma de terapia se basa en la búsqueda de la felicidad, lo que hace unos años no era una temática de estudio.

La terapia en base a la psicología positiva para pacientes con depresión tiene un enfoque derivado de la felicidad y el optimismo, obligando a las personas a pensar en forma positiva y no centrarse en las patologías, como se hace tradicionalmente en psicología. En el último tiempo Seligman ha ampliado el enfoque sobre el bienestar destacando la importancia de las relaciones sociales y el compro-miso (PERMA). En ese sentido nuestra intervención es coincidente en el fuerte énfasis que se a las relaciones interpersonales efectivas. A su vez, la psicología positiva propone un cambio de visión frente a las enfermedades, buscando la felicidad e incrementando las emociones positivas. De esta manera, se busca educar al paciente con el objetivo de hacer este cambio permanente, transformando el estilo cognitivo de las personas y, por tanto, disminuir las probabilidades de recaídas, las cuales son frecuentes en este trastorno (A. Cuadra et al., 2010; B. Vera, 2006).

Uno de los más efectivos elementos para prevenir y superar la depresión es una actitud positiva ante la vida, lo que se refleja en el nivel de satisfacción vital de las personas (Cuadra-Peralta, Veloso-Besio, Moya-Rubio, Reyes-Atabales, \& Vilca-Salinas, 2010). Diversos estudios encuentran bajo nivel de sintomatología depresiva en personas con alta satisfacción vital, además de otras características claramente asociadas a la depresión, como la calidad y cantidad de interacciones sociales y participación en diversas actividades, menos pensamientos negativos y más optimismo (Carneiro et al., 2007; Csikszentmihalyi \& Hunter, 2003; Diener \& Seligman, 2002; Tarlow, Schwartz, \& Haaga, 2002).

De acuerdo con todo lo mencionado anteriormente, el objetivo general del presente estudio es evaluar el impacto de un programa de intervención, basado en psicología positiva, sobre sintomatología depresiva y satisfacción vital, en adultos mayores.

\section{Psicología Positiva}

La exclusión de las emociones y aspectos positivos del ser humano como objeto de estudio y análisis de la psicología no se limita sólo al caso de la depresión, ya que la focalización tradicional y dominante de la psicología ha sido el estudio de la patología y la debilidad del ser humano, llegando a identificar y casi confundir psicología con psicopatología y psicoterapia (B. Vera, 2006). Esto ha desencadenado en una visión pesimista de la naturaleza humana (Gilham \& Seligman, 1999) que presupone la aceptación de un modelo que resta importancia a las características positivas del ser humano (Seligman \& Csikszentmihalyi, 2000). De esta manera, características como la alegría, el optimismo, la creatividad, el humor, la ilusión han sido ignoradas o explicadas superficialmente (H. Cuadra \& Florenzano, 2003).
En la última década, un grupo emergente de connotados investigadores (Csikszentmihalyi \& Hunter, 2003; Diener, Oishi, \& Lucas, 2003; Diener \& Seligman, 2002; Linley, Joseph, Harrington, \& Wood, 2006; M. Martínez, 2006; Park, Peterson, \& Seligman, 2004) se han fijado en estos olvidados aspectos del ser humano, tal como en la década de los 60 lo realizó la psicología humanística, al centrarse en los aspectos saludables y positivos del desarrollo humano. A diferencia de sus predecesores, estos investigadores han destacado por el fuerte sustento empírico que han brindado a sus planteamientos (Seligman et al., 2006). Este campo de estudio se denomina psicología positiva, agrupando la investigación de diversos factores que favorecen el desarrollo de la felicidad humana (Seligman et al., 2006).

La psicología positiva tiene por propósito mejorar la calidad de vida y prevenir la aparición de trastornos mentales y patologías, generando instancias que ayuden a la construcción de competencias que disminuyan la aparición de patologías, mejoren su pronóstico y, sobre todo, propicie el desarrollo del bienestar y felicidad de las personas (H. Cuadra \& Florenzano, 2003).

Ahora bien, la psicología positiva requiere de un constructo mensurable que facilite el desarrollo de sus investigaciones, es por esto que se conceptualiza y define la felicidad como el homólogo de bienestar subjetivo, el cual está compuesto por dos facetas básicas: una centrada en los aspectos afectivos-emocionales; estados de ánimo de la persona y la otra centrada en los aspectos cognitivosvalorativos; denominada satisfacción vital, siendo la evaluación personal sobre el bienestar en diferentes áreas de la vida (H. Cuadra \& Florenzano, 2003). Seligman (2003) plantea que para influir positivamente en ésta se deben identificar y cultivar las fortalezas más importantes de la persona y utilizarlas cotidianamente en el trabajo, el amor, el ocio y la educación de los hijos. Por lo tanto, cuando las personas aplican sus fortalezas durante su vida, logran desarrollar protección contra el infortunio, la depresión y las emociones negativas (A. Cuadra et al., 2010). Lo que conlleva que el rumbo de la vida se oriente hacia ámbitos más positivos, potenciando la creatividad, la tolerancia, la apertura a nuevas ideas y experiencias (Diener \& Seligman, 2002).

Con respecto a lo anterior, la felicidad o satisfacción vital y la salud de las personas son de primera relevancia hoy en día, lo cual ha sido avalado por los trabajos de los investigadores en psicología positiva (Danner, Snowdon, \& Friesen, 2001; Steptoe, Wright, Kunz-Ebrecht, \& Lliffe, 2006; Wong \& Fielding, 2007). Este enfoque teórico se ha ido posicionando en el estudio científico y el quehacer profesional, el cual se ha adoptado como una nueva y prometedora forma de tratamiento llamada psicoterapia positiva, que interviene sobre tres grandes aspectos que constituyen el concepto de felicidad: las emociones positivas (vida placentera), compromiso (una vida comprometida) y el significado (una vida con significado) (Seligman, 2003). 
Esta nueva forma de intervención ha mostrado resultados sorprendentes en el tratamiento de depresión superando, incluso, a los resultados obtenidos con psicoterapia cognitiva conductual. Ejemplo de ello son los resultados del estudio de Seligman et al. (2006), en que se comparó los efectos de la psicoterapia tradicional, terapia farmacológica y psicoterapia positiva, encontrándose que esta última fue la más eficaz en el tratamiento de la depresión. A su vez, en otro estudio, realizado en una muestra de pacientes diagnosticados con depresión, se encontró una mejoría significativa en la disminución de la depresión y un aumento significativo en la satisfacción vital de éstos, mediante la aplicación de sesiones grupales de psicoterapia positiva (A. Cuadra et al., 2010). Ambos estudios dan cuenta de la eficacia de esta intervención como tratamiento para la depresión.

La relevancia de desarrollar aspectos positivos del ser humano ha sido constatada empíricamente, encontrándose, por ejemplo, que el optimismo o pensamiento optimista ayuda a las personas a enfrentar de una mejor forma las situaciones adversas, por el contrario, las personas pesimistas hacen interpretaciones negativas, mostrando baja perseverancia frente a los obstáculos y desarrollando un elevado nivel de depresión (Puskar, Sereika, Lamb, Tusaire-Mumford, \& Mcguinness, 1999).

Otro aspecto positivo, cuyos beneficios han sido estudiados, es el rol que juega la gratitud en la satisfacción vital, demostrándose que las personas que experimentan elevada gratitud tienden a evaluar su propia vida con mayor satisfacción, lo cual es incompatible con los sentimientos experimentados en la depresión. Por lo tanto, la gratitud puede convertirse en una herramienta eficaz contra dicha patología (Watkins, Woodward, Stone, \& Kolts, 2003).

En la presente investigación, se ha realizado una adaptación de la psicoterapia positiva de Seligman, de acuerdo a la población específica y a las necesidades de los adultos mayores, con el objetivo de disminuir y/o prevenir la sintomatología depresiva y aumentar la satisfacción con la vida. Se toman en cuenta las diferentes técnicas utilizadas por la psicoterapia positiva, las cuales han sido sistematizadas en once sesiones, interviniendo en los tres aspectos de la satisfacción del ser humano.

Para fomentar la satisfacción vital, la psicoterapia positiva, se enfoca en desarrollar los siguientes aspectos del ser humano: satisfacción con el pasado, satisfacción con el presente y optimismo en el fututo (Seligman et al., 2006). Para lograr este objetivo se trabaja en sesiones grupales, donde se abordan las siguientes temáticas: las fortalezas, las emociones positivas, el perdón y olvido, la gratitud, las gratificaciones, el optimismo en el futuro, el disfrute de la vida, entre otras. Esta sistematización cuenta con fuertes y positivos resultados (Cuadra-Peralta et al., 2010; Seligman et al., 2006).

Para aumentar la satisfacción con el pasado, se interviene con las siguientes técnicas: la gratitud: la cual se refiere a ser consciente y agradecido de las cosas buenas que han sucedido en nuestra vida (Seligman, 2003); perdón y olvido: consiste en que una persona comprende racionalmente cuando ha sido tratada injustamente y es capaz de perdonar dejando atrás el resentimiento, respondiendo con principios de caridad, compasión, valor incondicional y amor (Enright \& Fitzgibbons, 2000, citados en Muñoz, Vinsonneau, Neto, Girard, \& Mullet, 2003). Por lo tanto, se considera este aspecto como fundamental para la satisfacción en el adulto mayor, lo cual ha sido avalado por diversas investigaciones revisadas por Muñoz et al. (2003), las que hacen referencia a cómo el perdonar aumenta la satisfacción y la salud.

Para fomentar la satisfacción con el presente, se utilizan las técnicas de: las bendiciones, la cual tiene como objetivo enseñar a las personas a agradecer día a día por las cosas simples de la vida (Seligman, Steen, Park, \& Peterson, 2005); el disfrute de la vida, que se enfoca en aumentar los placeres, potenciando lo que estamos realizando; y las gratificaciones las cuales se entienden como la sensación de bienestar que se experimenta al realizar una tarea (Seligman, 2003).

Con respecto a la satisfacción en el futuro, se considera al optimismo como un aspecto a fomentar, el cual es definido como una predisposición estable en creer que las cosas buenas suceden antes que las malas, entendiéndose como una dimensión continua, donde en un extremo se encuentra el pesimismo y, en el otro, el optimismo (Bryant \& Cvengros, 2004).

A su vez, las fortalezas juegan un rol importante dentro de las sesiones, ya que éstas influyen directamente en la satisfacción con la vida. Se ha establecido que existen 24 fortalezas, las cuales son disposiciones para actuar, decidir y sentir que implican el ejercicio de juzgar y guiar la conducta hacia la excelencia humana (M. Martínez, 2006; Peterson \& Seligman, 2004).

Finalmente, se incluyen las habilidades sociales. Esta técnica, según Pades (2003), cuenta con años y sustentada evidencia de efectividad, ya que no es ningún secreto, que millones de personas en la sociedad occidental actualmente no son felices en su vida social, por lo tanto, si no lo son en este ámbito, es poco probable que lo sean en su vida en general. Por ende, para lograr una mayor felicidad general tenemos que conseguir una mayor satisfacción en el área social. Para ello, se precisa de un conjunto de habilidades denominadas sociales (Caballo, 2000), las cuales son facilitadoras de emociones positivas (Fredickson, 2001), sustentado en éstas, los autores incorporaron técnicas basadas en las habilidades sociales, con especial atención en la asertividad, expresión de afecto y hacer cumplidos. Estos tópicos tienen como objetivo lograr una mayor inserción del adulto mayor en los contextos sociales, ya que el aislamiento social juega un papel importante en este segmento etáreo (M. Martínez, 2006), con claros efectos negativos como elevada presión arterial, obesidad, falta de actividad física y depresión, entre otros. Por el contrario, un buen nivel de interacciones 
sociales, y de buena calidad, se asocia a mejor salud y una vida más larga (Carneiro et al., 2007).

A partir de la bibliografía revisada y en función del propósito del presente estudio, las hipótesis a contrastar son las siguientes:

Hipótesis 1. La satisfacción vital mejorará significativamente sólo en el grupo de psicología positiva.

Hipótesis 2. La sintomatología depresiva disminuirá significativamente sólo en el grupo de psicología positiva.

\section{Método}

\section{Participantes}

Cuarenta y tres adultos mayores pertenecientes a agrupaciones de adultos mayores de la ciudad de Arica. Trece pertenecientes a la Agrupación de Adultos Mayores Escuela 18, los cuales conforman el grupo de talleres de psicología positiva (grupo cuasi experimental), con una edad promedio de 71 años $(S D=5.87)$; un $100 \%$ de este grupo fueron mujeres. Un segundo grupo, está compuesto por dieciséis adultos mayores, pertenecientes a la Agrupación de Adultos Mayores San Ignacio de Loyola, siendo éste el grupo de actividades recreativas (grupo cuasi control activo), con una edad promedio de 78 años ( $S D=$ 6.22 ), el cual está compuesto en un $100 \%$ por mujeres. Finalmente, el tercer grupo, que no recibe intervención (grupo cuasi control inactivo), está conformado por 14 adultos mayores pertenecientes a la Asociación de Jubilados Municipales, con una edad promedio de 74 años $(S D=8.26)$, del cual un $21.42 \%$ son hombres $(N=3)$, un $78.57 \%$ son mujeres $(N=11)$.

La técnica de muestreo utilizada es de carácter no probabilístico, por disponibilidad.

\section{Variables}

Variable Independiente. Programa de intervención, con tres niveles. Condición 1: grupo cuasi experimental, que recibe psicoterapia positiva; condición 2: grupo cuasi control activo, que recibe actividades recreativas; condición 3: cuasi control inactivo, no recibe intervención.

Variables Dependientes. Sintomatología Depresiva y Satisfacción Vital.

\section{Instrumentos}

Para evaluar sintomatología depresiva, se aplicó la Escala de Depresión Geriátrica de Yesavage, la cual consta de 30 ítems, con una escala dicotómica. Cada ítem mide un síntoma; por ejemplo: satisfacción con la vida, estado de ánimo, perspectiva de futuro, interacción social, entre otros. El instrumento tiene una consistencia interna de .78 y está validado en Chile (A. Cuadra, Arenas, \& Arratia, 1996).

Para medir Satisfacción Vital, se utilizó la Escala de Satisfacción Vital de Diener, Emmons, Larsen y Griffin (1985), el instrumento consta de cinco afirmaciones. El formato de respuesta corresponde a una escala de tipo Likert, con siete opciones de respuesta, que indica el gra- do de acuerdo con cada ítem, donde siete representa "estoy muy de acuerdo" y uno representa "estoy muy en desacuerdo". El instrumento posee una consistencia interna de .87, así como una consistencia test-retest de .82 .

\section{Diseño y Procedimiento}

Para conformar la muestra del estudio se contactaron asociaciones y agrupaciones que estuvieran dispuestas a formar parte del estudio. Luego, los investigadores se reunieron de manera directa con todos los adultos mayores que formaban parte de dichas agrupaciones y asociaciones y se les invitó a participar. Para resguardar el aspecto ético de la investigación, se entregó a cada uno de los adultos mayores una carta de consentimiento informado. Aquellos participantes que requirieron de una explicación adicional sobre dicha carta, se les proporcionó la información solicitada, enfatizando que la participación era de carácter voluntario y que podían abandonar el proceso cuando lo estimaran conveniente.

Se diseñó un manual y bitácora de actividades basadas en psicología positiva para ser desarrollados en un grupo de adultos mayores. Posteriormente se realizaron mediciones previas a la intervención del nivel de satisfacción vital y sintomatología depresiva en los tres grupos: grupo cuasi experimental de psicología positiva, grupo cuasi control activo de actividades recreativas y el grupo cuasi control inactivo.

A continuación se desarrollaron once sesiones de psicología positiva (primera introductoria, nueve correspondiente a la aplicación del manual y una final de cierre) a un grupo de adultos mayores pertenecientes a la Agrupación de Adultos Mayores Escuela 18.

La duración de los talleres para la condición uno (psicología positiva) fue de dos meses y dos semanas, con una intensidad media de dos horas semanales y una intensidad total de veintidós horas.

La duración de los talleres para la condición dos (actividades recreativas) fue de dos meses y dos semanas, con una intensidad media de una hora semanal y una intensidad total de once horas. Éstos fueron realizados por estudiantes de la carrera de Educación Física de la Universidad de Tarapacá y el objetivo de los talleres fue generar mayor movilidad física y distracción en este grupo.

La intervención fue grupal para ambos grupos cuasi control activo y cuasi experimental, con tipo de entrenamiento directo. Al grupo cuasi control inactivo no se le realizó intervención alguna.

Se realizó una medición posterior a la intervención del nivel de satisfacción vital y sintomatología depresiva en los tres grupos que conformaron la muestra, con la finalidad de determinar la eficacia de los talleres recibidos.

El diseño del presente estudio es cuasi experimental con mediciones pre y post intervención, con dos grupos cuasi control y un grupo cuasi experimental.

Los talleres en base a psicología positiva para adultos mayores se estructuraron en las siguientes sesiones: 
Tabla 1

Estructura de los Talleres

\begin{tabular}{lll}
$\mathrm{N}^{\mathrm{o}} \begin{array}{l}\text { Nombre } \\
\text { de la sesión }\end{array}$ & Descripción & Tarea \\
\hline
\end{tabular}

1 Presentación Presentación de las monitoras.

y Compromiso Introducción a la Psicología Positiva.

Breve descripción de los Talleres.

Finalmente, se realiza la aplicación de los

instrumentos: Cuestionario Sociodemográfico,

Escala de Satisfacción Vital y Cuestionario

de Depresión Geriátrica de Yesavage.

2 Satisfacción con el Placeres: se da una breve descripción y definición presente: Placer, Gratificaciones y Bendiciones

3 Fortalezas y Virtudes

4 Fortalezas y Virtudes

5 Relaciones interpersonales

6 Expresión de afecto y hacer cumplidos de éste. Se insta a los participantes a identificar y reconocer aquellas cosas que les son placenteras. Luego se enseñan técnicas para potenciar el placer experimentado.

Gratificaciones: se describe y define brevemente el concepto de gratificaciones. Se incita a los participantes a buscar aquellas actividades que les son gratificantes.

Bendiciones: se expone la importancia de las bendiciones y el rol que juega en la felicidad.

Revisión de la tarea asignada en la sesión anterior. Descripción del concepto de fortalezas y virtudes. Se dan a conocer las virtudes transversales a todas las culturas y las fortalezas correspondientes.

Aplicación y puntuación del Test de fortalezas VIA.

Revisión de la tarea asignada en la sesión anterior. Cada participante escoge dos fortalezas que reconozca como propias y trabaja en el desarrollo de éstas, buscando formas de potenciarlas.

Revisión de la tarea asignada en la sesión anterior. Se expone la importancia de las relaciones sociales, asimismo se analiza la influencia de éstas en las emociones positivas y negativas.

Se revisan formas de mejorar el tipo de comunicación.

Revisión de la tarea de la sesión anterior. La sesión se divide en dos partes.

Parte I: Expresión de amor, agrado y afecto. Se revisa la importancia de la expresión de afecto en las relaciones sociales y el rol que éste juega en la satisfacción de las personas. Se identifican diferentes formas de expresión de amor, agrado y afecto.

Parte II: Hacer y Recibir cumplidos. Se exponen la importancia, implicancia y consecuencias de hacer y recibir cumplidos. Se enseñan formas de hacer y recibir cumplidos.
Se le pide a los participantes escribir tres cosas buenas que le hayan pasado en el día, durante una semana., aplicando las técnicas pasadas para maximizar el disfrute.

Asimismo, se motiva a los asistentes a realizar al menos dos veces a la semana una actividad que les sea gratificante. Finalmente, se les pide que llenen en su casa una hoja de auto-registro, correspondiente a la sesión.

En base a los resultados obtenidos en el VIA, se solicita a los participantes buscar una persona que admiren y posea fortalezas similares a las suyas, luego deben anotar las características de ésta persona escogida.

Se insta a los participantes a ejercitar las fortalezas elegidas con un mínimo de tres veces por semana.

Después se pide que llenen una hoja de auto-registro, correspondiente a la sesión.

Se les pide a los participantes que identifiquen durante la semana situaciones sociales que faciliten afectos positivos y afectos negativos, para después rellenar la hoja de auto-registro correspondiente para cada sesión.

Los asistentes deben realizar cumplidos y expresar amor, agrado y afecto al menos a dos personas durante la semana. Después deben llenar una hoja de autoregistro. 
7 Satisfacción Revisión de la tarea de la sesión anterior. con el pasado: Perdón y Olvido

8 Satisfacción con el pasado:Perdón y Olvido

9 Optimismo sobre el futuro

10 Gratitud

\section{Resultados}

Se aplicaron pruebas $t$, para muestras relacionadas,

11 Taller de Revisión de la tarea de la sesión anterior.

Se comenta la experiencia de la tarea realizada.

Se hace un recuento de todos los contenidos revisados durante los Talleres. en cada uno de los grupos (ver Tabla 2). El objetivo es evaluar si los cambios observados son estadísticamente significativos.

Tabla 2

Diferencia de Medias en las Mediciones Pre y Post Intervención entre los Grupos

\begin{tabular}{|c|c|c|c|c|c|c|c|c|c|}
\hline & & \multicolumn{4}{|c|}{ Sintomatología Depresiva } & \multicolumn{4}{|c|}{ Nivel de Satisfacción Vital } \\
\hline & $N$ & $\begin{array}{c}\text { Pre } \\
\text { Media }\end{array}$ & $\begin{array}{c}\text { Post } \\
\text { Media }\end{array}$ & $T$ & $\rho$ & $\begin{array}{c}\text { Pre } \\
\text { Media }\end{array}$ & $\begin{array}{c}\text { Post } \\
\text { Media }\end{array}$ & $T$ & $\rho-$ \\
\hline Grupo Act. Recreativas & 16 & 13.83 & 12.38 & .92 & .36 & 26.22 & 28.37 & 1.05 & .30 \\
\hline Grupo Inactivo & 14 & 11.00 & 8.86 & .87 & .39 & 26.85 & 24.78 & .81 & .43 \\
\hline Grupo Positivo & 13 & 9.38 & 4.77 & 4.69 & .01 & 27.43 & 30.31 & 2.34 & .04 \\
\hline
\end{tabular}

En la Tabla 2, se puede observar las diferencias prepost intervención de los tres grupos que conforman la muestra. Al analizar los resultados se evidenciaron diferencias significativas en la sintomatología depresiva, sólo en el grupo de talleres de psicología positiva $[t(12)=4.69$, $p=.01]$. En cuanto al nivel de satisfacción vital se observó diferencias significativas sólo en el grupo de talleres de psicología positiva $[t(12)=2.334, p=.03]$.

Por último, en los otros dos grupos, no se evidenció diferencias significativas en el nivel de sintomatología
Los participantes deben identificar situaciones dolorosas que no han perdonado y desean perdonar. Luego deben rellenar una hoja de auto-registro correspondiente a la sesión.

Los asistentes deben completar una hoja de auto-registro, en base a la actividad desarrollada durante el taller.

Se insta a los participantes a buscar sucesos negativos durante la semana y cambiar el estilo atributivo a uno optimista. Luego deben llenar la hoja de autoregistro correspondiente a la sesión.

Se insta a los asistentes a que busque a una persona de la que estén muy agradecidos. Durante la semana deben escribirle una carta a dicha persona y leerla. 
respectiva. Esta técnica de análisis sirve para conocer el efecto de una variable independiente categórica (en este caso grupo de intervención) sobre una variable dependiente cuantitativa (depresión y satisfacción vital post) eliminando el efecto (parcializar) el efecto de otra variable cuantitativa (depresión y satisfacción vital pre).

El ANCOVA fue significativo para las dos variables dependientes. Para los indicadores de depresión se obtuvo que la prueba $\mathrm{F}$ resultó ser estadísticamente significativa, $F(2,40)=8.916, p=.001$. El tamaño del efecto medido con el eta parcializado al cuadrado fue $=.308$. Esto significa que el efecto grupo de intervención, afecta el 30.8 de la varianza de la medida post, una vez controlado el efecto de la medida pre (covariable). El grupo sin intervención tuvo un promedio ajustado de 9.193, el grupo de actividades recreativas tuvo una media ajustada de 11.306 y el grupo al cual se les aplicó la intervención en psicología positiva, su media ajustada fue 5.671 (entre más bajo, mejor, por ser un indicador de síntomas depresivos). El procedimiento de Holm Bonferroni fue utilizado para el control del error de tipo I en las tres comparaciones dos a dos, existiendo diferencias significativas del grupo positivo con los otros dos grupos.

Para la satisfacción vital, los valores obtenidos fueron $F=(2,40)=5.783, p=.006$ y eta cuadrado parcializada $=.224$, Las medias ajustadas fueron: grupo recreativo $=$ 28.383 , grupo control pasivo $=24.847$ y grupo psicología positiva $=31.358$, existiendo diferencias significativas del grupo positivo con los otros dos grupos.

\section{Conclusión y Discusión}

El objetivo inicial de este trabajo era medir y comprobar la eficacia de la aplicación de talleres basados en psicología positiva, como una innovadora forma de intervención en adultos mayores, para disminuir el nivel de sintomatología depresiva y aumentar la satisfacción vital. Los resultados evidenciaron que solamente en el grupo que asistió a los talleres de psicología positiva, en comparación al grupo cuasi experimental de actividades recreativas y al grupo cuasi control, se observó una variación estadísticamente significativa en el nivel de satisfacción vital y sintomatología depresiva.

Respecto de la satisfacción vital, los resultados obtenidos en la presente investigación confirman la efectividad del programa basado en psicología positiva, evidenciándose un aumento significativo sólo en el grupo que recibió los talleres de psicología positiva. Dichos resultados son congruentes con los encontrados en la investigación llevada a cabo por Seligman et al. (2006), donde los pacientes que recibieron psicoterapia positiva aumentaron significativamente su nivel de satisfacción vital.

Dentro de las implicancias de estos resultados, se puede encontrar en la literatura diversas investigaciones (Puskar et al., 1999; Steptoe et al., 2006) que dan cuenta cómo el aumento de la satisfacción vital y el aumento de las emo- ciones positivas amplían el repertorio conductual de las personas, convirtiéndolas en sujetos más positivos, flexibles, creativos, eficientes y abierto a la nueva información. De lo anterior se desprende que, las personas al tener un mayor repertorio conductual, mayor creatividad, flexibilidad, entre otras características, aumentan su capacidad de afrontar situaciones adversas de forma más exitosa, en comparación con personas que presentan mayormente emociones negativas (Fredickson, 2001).

Respecto de la segunda hipótesis de trabajo establecida, se encontró una disminución estadísticamente significativa, sólo en el grupo de talleres de psicología positiva, de la sintomatología depresiva, lo cual constituye una evidencia empírica a favor de la efectividad de la intervención en psicología positiva. Estos resultados son concordantes con el estudio de A. Cuadra et al. (2010) y con los resultados reportados por Seligman et al. (2006)

En base a lo expuesto anteriormente se puede concluir que la realización de talleres, basados en psicología positiva, es una forma de intervención eficaz para disminuir los síntomas depresivos y aumentar la satisfacción vital en la población de adultos mayores. Debido a que la intervención se focaliza, principalmente, en aumentar los tres grandes aspectos de la felicidad: la vida comprometida, una vida con significado y las emociones positivas, siendo estas últimas incompatibles con las emociones negativas, lo cual sugiere un efecto protector contra la depresión (Fredickson, 2001).

La presente investigación presentó algunas limitaciones debido a que el tipo de muestreo utilizado fue por disponibilidad, trabajándose con grupos formados previamente, con lo que no pudo asegurar la absoluta equivalencia inicial de las variables dependientes entre las tres condiciones. Así se tuvo que el grupo que recibió los talleres de psicología positiva tenía niveles un poco más bajos de sintomatología depresiva, en comparación a los otros dos grupos; también eran levemente más jóvenes, aunque ninguna de estas diferencias resultó estadísticamente significativa. No obstante lo anterior, los autores consideran que estas limitaciones no invalidan las conclusiones. El hecho de tener un nivel iniciales más bajo de sintomatología depresiva, constituye un obstáculo más que una ventaja, pues el margen de mejoría es más estrecho, que en personas con indicadores de depresión altos. Similar razonamiento es válido para la satisfacción vital, aunque inicialmente no había diferencias significativas entre los tres grupos, el que tenía mayor puntaje y, por ende, menos margen de mejoraría fue precisamente el grupo de intervención con psicología positiva.

Finalmente, un aspecto a destacar es que la aplicación de los talleres resulta eficiente debido al bajo costo de su implementación, por ser grupales y con relativamente pocas sesiones, por lo que su costo en tiempo y dinero es menor. Por otro lado, los talleres pueden ser llevados a cabo por alumnos egresados y/o con poca experiencia en el área clínica, ya que no se aplican técnicas que impliquen 
alta capacidad de contención emocional o el manejo de situaciones altamente dolorosas o conflictivas, lo que tiene la ventaja anexa de evitar la exposición pública de situaciones que muchos participantes prefieren mantener en reserva, disminuyendo la probabilidad de deserción de los talleres.

\section{Referencias}

Araujo, L., \& Lobo Filho, J. (2009). Análise psicossocial da violência contra idosos. Psicologia: Reflexão e Crítica, 22(1), 153-160. Retrieved from http://www.scielo.br/scielo.php? script $=$ sci_arttext\&pid $=$ S0102-79722009000100020\& lng=en\&nrm=iso\&tlng $=\mathrm{pt}$

Bryant, F., \& Cvengros, J., (2004). Distinguishing hope and optimism: Two sides of a coin, or two separate coins? Journal of Social and Clinical Psychology, 23(2), 273-302.

Caballo, V. (2000). Manual de evaluación y entrenamiento de las habilidades sociales. Madrid, España: Siglo Veintiuno.

Carneiro, R., Falcone, E., Clark, C., Del Prette, Z., \& Del Prette, A. (2007). Qualidade de vida, apoio social e depressão em idosos: Relação com habilidades sociais. Psicologia: Reflexão e Critica, 20(2), 229-237. Retrieved from http:// www.scielo.br/scielo.php?script=sci_arttext\&pid=S010279722007000200008\&lng=en\&nrm=iso\&tlng=pt

Contreras, D., Moreno, M., Martínez, M., Araya, P., Livacic, P., \& Vera, P. (2006). Efectos de una intervención cognitivo conductual sobre variables emocionales en adultos mayores. Revista Latinoamericana de Psicología, 38, 45-48.

Coon, D., \& Thompson, L. (2003). The relationship between homework compliance and treatmentment outcomes among older adult outpatients with mild-to-moderate depresión. American Journal of Geriatric Psychiatry, 11, 53-61.

Csikszentmihalyi, M., \& Hunter, J. (2003). Happiness in everyday life: The uses of experience sampling. Journal of Happiness Studies, 4, 185-199.

Cuadra, A., Arenas, M., \& Arratia, R. (1996). Adaptación y validación del Geriatric Depression Scale (GDS para adultos mayores del gran Santiago). (Unpublished doctoral dissertation). Universidad de Tarapacá, Arica, Chile.

Cuadra, A., Veloso, C., Ibergaray, M., \& Rocha, M. (2010). Resultados de la psicoterapia positiva en pacientes con depresión. Terapia Psicológica, 20(1), 127-134. Retrieved from http://www.scielo.cl/scielo.php?script=sci_arttext\& pid=S0718-48082010000100012\&lng=es\&nrm=iso\&tlng=es

Cuadra, H., \& Florenzano, R. (2003). El bienestar subjetivo: hacia una psicología positiva. Revista de Psicología de la Universidad de Chile, 13(1), 83-96.

Cuadra-Peralta, A., Veloso-Besio, C., Moya-Rubio, Y., ReyesAtabales, L., \& Vilca-Salinas, J. (2010). Efecto de un programa de psicología positiva e inteligencia emocional sobre la satisfacción laboral y vital. Revista Salud \& Sociedad, 1(2). Retrieved from http://www.saludysociedad.cl/index.php/ main/article/view/17/37

Danner, D., Snowdon, D., \& Friesen, W. (2001). Positive emotions in early life and longevity findings from the nun study. Journal of Personality and Social Psychology, 80(5), 804-813.

Departamento de Estadística e Información de Salud. (2006). II Encuesta de Calidad de Vida y Salud. Santiago, Chile: Autor.
Diener, E., Emmons, R. A., Larsen, R. J., \& Griffin, S. (1985). The Satisfaction with Life Scale. Journal of Personality Assessment, 49(1), 71-75.

Diener, E., Oishi, S., \& Lucas, R. (2003). Personality, culture, and subjective well-being: Emotional and cognitive evaluations of life. Annual Review of Psychology, 54, 403-425.

Diener, E., \& Seligman, M. (2002). Very happy people. Psychological Science, 13(1), 81-84.

Enright, R., \& Fitzgibbons, R. (2000). Helping clients forgive: An empirical guide for resolving anger and restoring hope. Washington, DC: American Psychological Association.

Fredickson, B. (2001). The role of positive emotions in Positive Psychology. American Psychologist, 53(3), 218-226.

Gilham, J., \& Seligman, M. (1999). Footsteps on the road to a Positive Psychology. Behavior Research and Therapy, 37, 163-173.

Instituto Nacional de Estadísticas. (2002). Censo de población $y$ vivienda, Chile. Resultados generales, 2002. Santiago, Chile: Autor.

Linley, P., Joseph, S., Harrington, S., \& Wood, A. (2006). Positive Psychology: Past, present, and (possible) future. The Journal of Positive Psychology, 1(1), 3-16.

Martín, M., Baca, E., Alvarez, E., Bousoño, M., Eguiluz, I., Roca, M., et al. (2008). Depresión a largo plazo en pacientes geriátricos. Actas Españolas de Psiquiatría, 62-68.

Martínez, J., Onís, M., Dueñas, R., Albert, C., Aguado, C., \& Luque, R. (2002). Versión española del Cuestionario de Yesavage Abreviado (GDS) para el despistaje de depresión en mayores de 65 años: adaptación y validación. Medifam, 12, 620-630.

Martínez, M. (2006). El estudio científico de las fortalezas trascendentales desde la psicología positiva. Clínica y Salud, 17(3), 245-248.

Muñoz, M., Vinsonneau, G., Neto F., Girard M., \& Mullet E. (2003). Forgivingness and satisfaction with life. Journal of Happiness Studies, 4, 323-335.

Pades, A. (2003) Habilidades sociales en enfermería: propuesta de un programa de intervención. (Unpublished doctoral dissertation). Universitat de Les Illes Ballears, Palma, España. Retrieved from http://tdx.cat/bitstream/handle/10803/9444/ tapj1de1.pdf?sequence $=1$

Park, N., Peterson, C., \& Seligman, M. (2004). Strengths of character and well-being. Journal of Social and Clinical Psychology, 23(5), 603-619.

Pérez, M., \& García, J. (2001). Tratamientos eficaces para la depresión. Psicothema, 13(3), 493-510.

Peterson, C., \& Seligman, M. (2004). Character strengths and virtues: A handbook and classification. Oxford, UK: Oxford University Press.

Puskar, K., Sereika, S., Lamb, J., Tusaire-Mumford, K., \& Mcguinness, T. (1999). Optimism and its relationship to depression, coping, anger, and life events in rural adolescents. Issues in Mental Health Nursing, 20, 115-130.

Seligman, M. (2003). La auténtica felicidad. Madrid, España: Ediciones B.

Seligman, M., \& Csikszentmihalyi, M. (2000). Positive Psychology: An introduction. American Psychologist, 55, 5-14.

Seligman, M., Rashid, T., \& Parks, A. (2006). Positive Psychoterapy. American Psychologist, 774-786.

Seligman, M., Steen, T., Park, N., \& Peterson, C. (2005). Positive Psychology progress: Empirical validation of interventions. American Psychologist, 60(5), 410-421. 
Steptoe, A., Wright, C., Kunz-Ebrecht, S., \& Lliffe, C. (2006). Dispositional optimism and Elath behaviour in communitydwelling older people: Associations with healthy ageing. British Journal of Health Psychology, 11, 71-84.

Tarlow, E., Schwartz, M., \& Haaga, D. (2002). Are the very happy too happy? Journal of Happiness Studies, 3, 355-372.

Vera, B. (2006). Psicología positiva: una nueva forma de entender la psicología. Papeles del Psicólogo, 27, 3-8.

Watkins, P., Woodward, K., Stone, T., \& Kolts, R. (2003). Gratitude and happiness: Development of a measure of gratitude, and relationships with subjetive well-being. Social Behavior and Personality, 491-452.

Wong, W., \& Fielding, R. (2007). Quality of life and pain in Chinese lung cancer patients: Is optimism a moderator or mediator? Quality of Life Research, 16, 53-63. 